\title{
The belief that secondhand smoke causes serious illness among Chinese smokers: Smoking cessation and intention to quit
}

\author{
Zachary Joseph Madewell
}

\begin{abstract}
INTRODUCTION Approximately 70\% of Chinese adults are exposed to secondhand smoke (SHS) each week and 100000 people die from SHS every year in China. This study evaluates associations between the belief that SHS causes serious illness and intention to quit, attempts to quit, and quitting smoking, among Chinese adult smokers.

METHODS A nationally representative sample of 4866 current and former adult smokers in the Global Adult Tobacco Survey was used for analysis. Multivariable weighted regression models were built to determine significant associations between smoking cessation behavior and the belief that SHS causes serious illness.

RESULTS The belief that SHS causes serious illness was associated with intention to quit (AOR 1.62, 95\% CI: 1.24, 2.12) and quitting smoking (AOR 1.44, 95\% CI: 1.15, 1.81). Other variables associated with smoking cessation behavior included not permitting smoking at home (intending: AOR 1.59, 95\% CI: 1.10, 2.31; attempting: AOR 1.73, 95\% CI: 1.25, 2.40; quitting: AOR 2.71, 95\% CI: $1.90,3.89$ ) and the belief that smoking causes serious illness (attempting: AOR 1.63, 95\% CI: 1.14, 2.33; quitting: AOR 1.66, 95\% CI: 1.21, 2.28).

CONCLUSIONS These results indicate that believing SHS causes serious illness may play a role in quitting smoking. In China's collectivistic culture, interventions should focus on how SHS exposure affects the health of friends and family. This message can be combined with other proven tobacco control methods such as: smoking bans in public places, warning labels on cigarette packages, high cigarette taxes, and mass media campaigns to reduce tobacco use.
\end{abstract}

AFFILIATION

1 University of California, San Diego, United States

CORRESPONDENCE TO

Zachary Joseph Madewell.

University of California, San

Diego, 9500 Gilman Dr, 92093

La Jolla, CA, United States.

E-mail: zmadewel@ucsd.edu

KEYWORDS

smoking cessation, secondhand smoke, China

Received: 15 September 2017

Revised: 7 January 2018

Accepted: 12 January 2018

\section{INTRODUCTION}

China is the world's largest producer, manufacturer, and consumer of tobacco ${ }^{1,2}$. Chinese men consume over one-third of the world's cigarettes ${ }^{3,4}$. The prevalence of smoking in China among people 15 years or older was $28.1 \%$ in 2010 , including $52.9 \%$ of men and $2.4 \%$ of women ${ }^{2}$. Although the quit rate has increased from $3 \%$ in 1991 to $9 \%$ in 2006 , one-third of male smokers will eventually die from tobaccorelated disease ${ }^{3}$.

Each year, exposure to secondhand smoke (SHS) causes over 435000 adult and 165000 childhood deaths globally ${ }^{5}$. Worldwide, $40 \%$ of children, $35 \%$ of women, and $33 \%$ of men are exposed to SHS indoors ${ }^{5}$. Adverse health outcomes including ischemic heart disease, lower respiratory infections, asthma, and lung cancer may be reduced with interventions limiting SHS exposure ${ }^{5}$.

Belief that SHS causes serious illness, and that it is associated with smoking behavior, has been studied extensively in Western nations ${ }^{6,7}$. The belief that SHS causes illness, particularly in children, is positively associated with quit attempts among smokers ${ }^{6,7}$. In Australia, one in five smokers attempted to quit due 
to concerns that their behavior was causing problems among friends and family ${ }^{6}$.

There is less information in China about the associations between adults' belief that SHS causes serious illness, and behavioral intention to quit, attempts to quit, and quitting smoking. Approximately $70 \%$ of adults are exposed to SHS each week and 100 000 people die from SHS every year in $\mathrm{China}^{8}$. Of all Chinese adults, $54.3 \%$ reported being exposed to SHS at work, $76.3 \%$ at restaurants, and $16.4 \%$ on public transportation ${ }^{9}$. Lower levels of income and education are associated with higher exposure to SHS in China ${ }^{10}$.

Positive associations were demonstrated between the awareness of personal health risks of smoking, and quit-smoking behavior ${ }^{11,12}$. Results from China's International Tobacco Control survey indicate pressure from friends and family motivate Chinese adults to attempt to quit smoking ${ }^{11,12}$. Health concerns for oneself and one's family were also cited as a primary reason for attempting to quit smoking in a study of male smokers in Hangzhou ${ }^{13}$. It is unclear, however, whether personal health concerns or SHS were the primary motivator for attempting to quit. It remains to be determined whether knowledge that SHS can harm friends and family has an impact on successfully quitting smoking. Described herein is a cross-sectional study of current Chinese smokers, age 15 years and older, surveyed in 2010 , to evaluate the associations between the belief that SHS causes serious illness and intention to quit, attempts to quit, and quitting smoking tobacco.

\section{METHODS}

\section{Questionnaire}

The World Health Organization (WHO) and US Centers for Disease Control and Prevention (CDC) launched the Global Adult Tobacco Survey (GATS) in 2007. GATS uses a standardized questionnaire to measure and track smoking, and key tobacco control indicators, in 25 low and middle income countries (LMIC), that account for over half of the world's adult population and smokers ${ }^{2,4,14,15}$. Results from GATS are used to implement tobacco control, prevention measures, and to make comparisons with other countries ${ }^{2}$.

GATSChina, a cross-sectional, nationally representative survey, was completed in 2010 and included: population demographics, tobacco usage, quitting behaviors, SHS exposures, economics; and knowledge, attitudes and perceptions about tobacco use ${ }^{2}$. GATS China included males and females age 15 years and older, excluding those living in dormitories, barracks, prisons, nursing homes, or hospitals ${ }^{2,4}$. Details of the interview, and the data compilation process, are available elsewhere ${ }^{2,4}$.

\section{Study population}

Stratified multi-stage cluster sampling was used, with probability-proportional-to-size random methods, for household selection. The first stage included urban districts or rural counties, the second stage identified neighborhood communities or villages, and the third stage selected groups of households at random ${ }^{2}$. One person was selected randomly from each household to participate in the survey ${ }^{2}$.

GATS China aimed at a sample size of 15000 participants, representative of China's six geographic regions ${ }^{2}$, each of which were further stratified into urban and rural areas ${ }^{4}$. Following exclusion criteria, 13562 household interviews were completed, with an overall household response rate of $97.5 \%{ }^{2}$. With an individual-level response rate of $98.5 \%, 13354$ nationally representative men and women age 15 years and older completed the interview in 2010, with 5832 and 7522 participants from urban and rural areas, respectively ${ }^{2}$. There were 4866 eversmokers (current and former smokers) included for analyses of quitting smoking ${ }^{2}$. Analyses of attempts and intention to quit smoking included 4010 current smokers.

\section{Variables}

The outcome variables for this study included 'ever intended to quit smoking' (yes, no), 'attempted to stop smoking' (yes, no), and 'quit smoking' (current smoker, former smoker). 'Intended to quit' included 'within the next month', 'within the next year', and 'someday but not within the next year'. 'Current' smokers were those who smoked any tobacco product on a daily or less than daily basis. 'Former' smokers were those who, in the past, had smoked any tobacco product daily.

The key exposure variable was 'belief that breathing smoke from other people's cigarettes causes serious illness in non-smokers (yes, no)'.

Covariates included 'believed smoking causes serious illness, stroke, heart disease, or cancer' (yes, no). 'No' also included 'do not know'. Other covariates were 'had seen or heard information about 
the dangers of smoking in newspapers, magazines, television, billboards, posters, promotional materials, public transportation vehicles, stations, or somewhere else' (yes, no). 'Somewhere else' included radio, public walls, cinemas, internet, or elsewhere. Another covariate was 'had noticed any advertisements or signs promoting cigarettes anywhere' (yes, no). 'Anywhere' included point of sale, television, radio, billboards, posters, promotional materials, newspapers, magazines, cinemas, internet, public transportation vehicles, stations, public walls, or elsewhere. Other variables were 'visited a doctor or another healthcare provider in the last 12 months' (yes, no) and 'the rule about smoking at home' (allowed, not allowed). 'Allowed' also included 'no rules', and 'don't know'. 'Not allowed' included 'never allowed', and 'not allowed, but exceptions'.

Additional covariates were gender, age group (15-24, 25-44, 45-64, 65+), highest level of education completed (no formal schooling, completed primary, completed secondary, university or higher), employment status over the previous 12 months (agriculture/forestry/ fishery employee, transportation/equipment operator, business/service industry employee, other occupation, unemployed), residence (urban, rural), region (North, North-East, East, Mid-South, South-West, North-West).

\section{Analysis}

Descriptive frequencies are reported for current smokers and ever-smokers. Odds ratio (OR) was used to evaluate the magnitude of the associations between exposures of interest (beliefs that smoking or SHS causes illness, stroke, lung cancer, or heart attack; noticed smoking warnings in newspapers, television, billboards, posters, public transportation, or elsewhere; noticed signs promoting cigarettes; the rule about smoking at home; doctor visits; gender; age; education; employment; residence; and region) and outcomes (intention to quit, attempts to quit, and quitting smoking).

Statistical significance was evaluated through the Wald chi-square test. Variables found to be significant at $p<0.10$ from bivariate analyses were included in step-wise multivariable logistic regression models to evaluate associations with the outcomes of interest (intention to quit, attempts to quit, and quitting smoking). Variables associated with outcomes at $\mathrm{p}<0.05$, as well as the main exposure, the belief that
SHS causes serious illness, were retained in the final models. The order of variables included in models was determined by significance. After significant variables were entered into the models from bivariate analyses, interaction terms cited in the literature were assessed, including the belief that SHS causes serious illness by gender, education, and home smoking rules ${ }^{2,16,17}$. Pairwise correlation coefficients, tolerance values, and the condition number (CN) were examined to assess interrelationships among covariates similar in construct for collinearity ${ }^{18}$. Adjusted ORs and 95\% Confidence Intervals (CIs) are reported. All analyses were calculated using SAS V.9.4 (SAS Institute, Inc., Cary, North Carolina). The surveyfreq and surveylogistic procedures were used to account for cluster, strata, and weight factors.

\section{RESULTS}

Of the nationally representative sample of 13354 men and women in China in 2010, current smokers were 4010 and former smokers were 856. After accounting for cluster, strata, and weight factors, this represents $28.1 \%$ as current smokers and $5.4 \%$ as former smokers (Table 1). Correlation matrices revealed no highly correlated interrelationships among any of the covariates examined, the tolerance values were well above 0.10 , and the $\mathrm{CN}=3.36$ was far below the rule-of-thumb of 30 (not tabulated) ${ }^{18}$. Therefore, there was no evidence of collinearity.

\section{Intended to quit}

Variables associated with intention to quit from bivariate analyses at $p<0.10$ included the belief that: SHS causes serious illness $(p<0.001)$; the belief that smoking causes serious illness $(\mathrm{p}<0.001)$ or lung cancer $(p=0.001)$; home smoking rules $(p=0.005)$; noticed information about the dangers of smoking on billboards $(p=0.022)$, on television $(p=0.020)$, or on public vehicles $(\mathrm{p}<0.001)$; noticed signs promoting cigarettes $(\mathrm{p}=0.018)$; age $(\mathrm{p}<0.001)$; and education $(\mathrm{p}=0.001)$ (Table 2).

The final model for current smokers intending to quit smoking included the belief that SHS causes serious illness (AOR: 1.62; 95\% CI: 1.24, 2.12, Table 2 ), the belief that smoking causes lung cancer (AOR: 1.36, 95\% CI: $1.00,1.83$ ), and rules not allowing smoking at home (AOR: 1.59, 95\% CI: 1.10, 2.31). Interaction terms were not significant at $\mathrm{p}<0.05$. 
Table 1. Descriptive statistics ${ }^{\mathrm{a}}$ of current and former smokers, 2010 China Global Adult Tobacco Survey

\begin{tabular}{|c|c|c|}
\hline Characteristic & $\begin{array}{c}\text { Current } \\
\text { smokers } \\
\text { Proportion } \\
\% \\
\text { (Std Err } \\
\%)\end{array}$ & $\begin{array}{c}\text { Former } \\
\text { smokers } \\
\text { Proportion } \\
\% \\
\text { (Std Err } \\
\%)\end{array}$ \\
\hline
\end{tabular}

Believe SHS causes illness

$\begin{array}{lll}\text { Yes } & 59.4(2.3) & 67.0(2.7) \\ \text { No } & 40.6(2.3) & 33.0(2.7)\end{array}$

Believe smoking causes Serious illness

Yes

$79.7(1.6) \quad 86.7(2.0)$

No

$20.3(1.6) \quad 13.3(2.0)$

Lung cancer

Yes

No

Stroke

Yes

No

Heart attacks

Yes

No

$\begin{array}{ll}42.5(1.8) & 48.7(3.0) \\ 57.5(1.8) & 51.3(3.0)\end{array}$

Rule about smoking at home

Not allowed

$12.7(1.4) \quad 27.2(3.5)$

Allowed

$87.3(1.4) \quad 72.8(3.5)$

Noticed smoking warnings (ref: no)

In newspapers

Yes

No

$22.1(2.0) \quad 23.4(2.6)$

On billboards

Yes

No

$81.8(1.6) \quad 82.0(2.2)$

$18.2(1.6) \quad 18.0(2.2)$

32.2 (1.9) $36.4(2.7)$

$67.8(1.9) \quad 63.6(2.7)$

$42.5(1.8) \quad 48.7(3.0)$

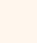

$22.1(2.0) \quad 23.4(2.6)$

$77.9(2.0) \quad 76.6(2.6)$

$20.6(2.2) \quad 19.8(2.3)$

$79.4(2.2) \quad 80.2(2.3)$

On television

Yes

No

46.8 (2.9) 47.9 (2.9)

53.2 (2.9) $52.1(2.9)$

On posters

Yes

No

$\begin{array}{cc}9.7(1.5) & 8.8(1.5) \\ 90.3(1.5) & 91.2(1.5) \\ & \\ 21.0(2.4) & 17.9(2.3) \\ 79.0(2.4) & 82.1(2.3)\end{array}$

$15.6(1.7) \quad 15.0(2.5)$ $84.4(1.7) \quad 85.0(2.5)$

Noticed cigarette promotions

Yes

$19.1(2.0) \quad 12.8(1.8)$
Table 1. Continued

\begin{tabular}{|c|c|c|}
\hline Characteristic & $\begin{array}{c}\text { Current } \\
\text { smokers } \\
\text { Proportion } \\
\% \\
\text { (Std Err } \\
\%)\end{array}$ & $\begin{array}{c}\text { Former } \\
\text { smokers } \\
\text { Proportion } \\
\% \\
\text { (Std Err } \\
\% \text { o) }\end{array}$ \\
\hline No & $80.9(2.0)$ & $87.2(1.8)$ \\
\hline \multicolumn{3}{|c|}{ Visited a doctor in past year } \\
\hline Yes & $29.2(1.8)$ & - \\
\hline No & $70.8(1.8)$ & - \\
\hline \multicolumn{3}{|l|}{ Gender } \\
\hline Male & $95.8(0.5)$ & $93.4(1.0)$ \\
\hline Female & $4.2(0.5)$ & $6.6(1.0)$ \\
\hline \multicolumn{3}{|l|}{ Age } \\
\hline $15-24$ & $10.9(1.4)$ & $5.8(1.9)$ \\
\hline $25-44$ & $42.4(1.7)$ & $24.3(2.3)$ \\
\hline $45-64$ & $37.6(1.3)$ & $41.7(3.0)$ \\
\hline $65+$ & $9.1(0.6)$ & $28.2(2.6)$ \\
\hline \multicolumn{3}{|l|}{ Education } \\
\hline$<$ Primary & $11.8(1.1)$ & $17.8(2.0)$ \\
\hline$<$ Secondary & $18.6(1.6)$ & $23.7(2.2)$ \\
\hline Secondary & $59.8(2.0)$ & $49.4(2.7)$ \\
\hline College & $9.8(1.2)$ & $9.1(1.6)$ \\
\hline \multicolumn{3}{|l|}{ Employment } \\
\hline Unemployed & $12.0(1.3)$ & $32.5(3.4)$ \\
\hline AFF & $34.5(4.0)$ & $31.0(4.2)$ \\
\hline Transportation & $19.6(1.9)$ & $8.9(1.7)$ \\
\hline Business/service & $16.5(1.6)$ & $10.2(1.5)$ \\
\hline Other & $17.4(1.7)$ & $17.4(3.1)$ \\
\hline \multicolumn{3}{|l|}{ Residence } \\
\hline Rural & $57.2(5.5)$ & $54.6(5.5)$ \\
\hline Urban & $42.8(5.5)$ & $45.4(5.5)$ \\
\hline \multicolumn{3}{|l|}{ Region } \\
\hline North & $13.1(2.0)$ & $8.4(1.5)$ \\
\hline North-East & $10.7(0.9)$ & $19.0(2.9)$ \\
\hline East & $28.0(3.0)$ & $32.2(4.1)$ \\
\hline Mid-South & $17.8(1.7)$ & $17.1(2.1)$ \\
\hline South-West & $23.7(2.3)$ & $17.7(4.2)$ \\
\hline North-West & $6.7(1.6)$ & $5.6(1.8)$ \\
\hline
\end{tabular}

SHS: secondhand smoke; AFF: agriculture, forestry, fishing

a Sample weights were used to account for clustering and stratification factors ${ }^{b}$ Collected for current smokers only

\section{Attempted to quit}

Variables associated with attempts to quit from bivariate analyses at $\mathrm{p}<0.10$ included the belief that smoking causes serious illness $(p=0.004)$ and heart attacks 
Table 2. Intended to quit smoking: unadjusted and adjusted associations ${ }^{a}$ with the belief that secondhand smoke causes serious illness among current smokers, 2010 China Global Adult Tobacco Survey

\begin{tabular}{|c|c|c|c|c|c|}
\hline Characteristic & $\begin{array}{c}\text { Intended } \\
\text { to quit } \\
\text { prevalence } \\
\% \\
\text { (Std Err \%) }\end{array}$ & $\begin{array}{l}\text { Unadjusted OR } \\
\qquad(95 \% \mathrm{CI})\end{array}$ & p value & $\begin{array}{l}\text { Adjusted }{ }^{b} \text { OR } \\
\qquad(95 \% \text { CII })\end{array}$ & p-value \\
\hline Believe SHS causes illness (ref: no) & $46.8(2.9)$ & $1.79(1.40,2.29)$ & $<0.001$ & $1.62(1.24,2.12)$ & $<0.001$ \\
\hline \multicolumn{6}{|l|}{ Believe smoking causes (ref: no) } \\
\hline Serious illness & $45.6(2.6)$ & $2.67(2.07,3.45)$ & $<0.001$ & - & - \\
\hline Lung cancer & $43.3(2.6)$ & $1.65(1.23,2.20)$ & 0.001 & $1.36(1.00,1.83)$ & 0.044 \\
\hline Stroke & $41.6(3.6)$ & $1.02(0.75,1.41)$ & 0.882 & - & - \\
\hline Heart attacks & $43.2(3.2)$ & $1.15(0.89,1.49)$ & 0.283 & - & - \\
\hline Smoking not allowed at home (ref: allowed) & $53.2(4.2)$ & $1.74(1.18,2.57)$ & 0.005 & $1.59(1.10,2.31)$ & 0.013 \\
\hline \multicolumn{6}{|l|}{ Noticed smoking warnings (ref: no) } \\
\hline In newspapers & $45.5(3.7)$ & $1.25(0.92,1.70)$ & 0.149 & - & - \\
\hline On billboards & $48.1(4.2)$ & $1.43(1.05,1.94)$ & 0.022 & - & - \\
\hline On television & $44.6(2.6)$ & $1.30(1.04,1.62)$ & 0.020 & - & - \\
\hline On posters & $45.4(5.9)$ & $1.21(0.78,1.87)$ & 0.388 & - & - \\
\hline On vehicles & $49.6(3.1)$ & $1.54(1.23,1.93)$ & $<0.001$ & - & - \\
\hline Elsewhere & $48.2(6.5)$ & $1.40(0.89,2.21)$ & 0.140 & - & - \\
\hline Noticed cigarette promotions (ref: no) & $48.2(4.5)$ & $1.42(1.06,1.91)$ & 0.018 & - & - \\
\hline Visited a doctor in past year (ref: no) & $43.6(2.1)$ & $1.15(0.92,1.44)$ & 0.225 & - & - \\
\hline Male (ref: female) & $41.5(2.3)$ & $1.39(0.90,2.17)$ & 0.136 & - & - \\
\hline Age (ref: 15-24) & & & $<0.001$ & & - \\
\hline $25-44$ & $44.4(3.2)$ & $1.32(0.75,2.30)$ & & - & \\
\hline $45-64$ & $41.3(2.6)$ & $1.16(0.72,1.88)$ & & - & \\
\hline $65+$ & $30.2(2.9)$ & $0.71(0.41,1.25)$ & & - & \\
\hline Education (ref: <primary) & & & 0.001 & & - \\
\hline$<$ Secondary & $38.3(2.4)$ & $1.32(0.97,1.80)$ & & - & \\
\hline Secondary & $44.5(3.0)$ & $1.71(1.31,2.24)$ & & - & \\
\hline College & $37.8(3.8)$ & $1.29(0.89,1.88)$ & & - & \\
\hline Employment (ref: unemployed) & & & 0.144 & & - \\
\hline AFF & $43.0(4.7)$ & $1.10(0.76,1.60)$ & & - & \\
\hline Transportation & $38.4(2.2)$ & $0.91(0.66,1.26)$ & & - & \\
\hline Business/service & $49.1(4.7)$ & $1.41(1.00,1.98)$ & & - & \\
\hline Other & $37.0(3.4)$ & $0.86(0.61,1.21)$ & & - & \\
\hline Rural residence (ref: urban) & $41.1(2.5)$ & $0.99(0.68,1.42)$ & 0.941 & - & - \\
\hline Region (ref: South-West) & & & 0.954 & & - \\
\hline North & $43.2(4.7)$ & $0.84(0.45,1.57)$ & & - & \\
\hline North-East & $39.0(4.3)$ & $0.90(0.53,1.53)$ & & - & \\
\hline East & $38.9(5.6)$ & $1.00(0.57,1.76)$ & & - & \\
\hline Mid-South & 40.7 (3.9) & $0.84(0.48,1.46)$ & & - & \\
\hline North-West & $44.5(5.8)$ & $1.06(0.56,1.97)$ & & - & \\
\hline
\end{tabular}

( $\mathrm{p}=0.092)$, home smoking rules $(\mathrm{p}<0.001)$, noticed information about the dangers of smoking in newspapers $(\mathrm{p}=0.016)$ or on billboards $(\mathrm{p}=0.001)$, visited a doctor $(\mathrm{p}=0.010)$, and age $(\mathrm{p}=0.065)($ Table 3$)$.

The final model for current smokers attempting to quit smoking included the belief that SHS causes 
Table 3. Attempted to quit smoking: unadjusted and adjusted associations ${ }^{\mathrm{a}}$ with the belief that secondhand smoke causes serious illness among current smokers, 2010 China Global Adult Tobacco Survey

\begin{tabular}{|c|c|c|c|c|c|}
\hline Characteristic & $\begin{array}{c}\text { Attempted } \\
\text { to quit } \\
\text { prevalence } \\
\% \\
\text { (Std Err \%) }\end{array}$ & $\begin{array}{l}\text { Unadjusted OR } \\
\qquad\left(95^{\circ} \% \mathrm{CI}\right)\end{array}$ & p-value & $\begin{array}{l}\text { Adjusted }{ }^{b} \text { OR } \\
\qquad(95 \% \text { CI })\end{array}$ & p value \\
\hline Believe SHS causes illness (ref: no) & $38.0(2.7)$ & $1.05(0.79,1.40)$ & 0.745 & $0.84(0.60,1.16)$ & 0.282 \\
\hline \multicolumn{6}{|l|}{ Believe smoking causes (ref: no) } \\
\hline Serious illness & $39.6(2.3)$ & $1.58(1.15,2.16)$ & 0.004 & $1.63(1.14,2.33)$ & 0.007 \\
\hline Lung cancer & $38.0(2.2)$ & $1.13(0.85,1.52)$ & 0.398 & - & - \\
\hline Stroke & $38.8(2.6)$ & $1.09(0.84,1.40)$ & 0.522 & - & - \\
\hline Heart attacks & $40.0(2.7)$ & $1.20(0.97,1.49)$ & 0.092 & - & - \\
\hline Smoking not allowed at home (ref: allowed) & $50.2(3.5)$ & $1.82(1.32,2.50)$ & $<0.001$ & $1.73(1.25,2.40)$ & 0.001 \\
\hline \multicolumn{6}{|l|}{ Noticed smoking warnings (ref: no) } \\
\hline In newspapers & $42.8(2.5)$ & $1.33(1.05,1.68)$ & 0.016 & - & - \\
\hline On billboards & $44.6(2.8)$ & $1.45(1.15,1.83)$ & 0.001 & $1.34(1.03,1.75)$ & 0.026 \\
\hline On television & $39.7(1.9)$ & $1.19(0.90,1.57)$ & 0.225 & - & - \\
\hline On posters & $40.8(4.1)$ & $1.16(0.82,1.64)$ & 0.387 & - & - \\
\hline On vehicles & $37.0(3.4)$ & $0.97(0.72,1.31)$ & 0.860 & - & - \\
\hline Elsewhere & $38.9(4.0)$ & $1.07(0.77,1.50)$ & 0.675 & - & - \\
\hline Noticed cigarette promotions (ref: no) & $35.7(3.2)$ & $0.91(0.68,1.22)$ & 0.515 & - & - \\
\hline Visited a doctor in past year (ref: no) & $43.6(2.6)$ & $1.43(1.09,1.88)$ & 0.010 & $1.46(1.12,1.90)$ & 0.005 \\
\hline Male (ref: female) & $37.6(2.0)$ & $1.06(0.71,1.59)$ & 0.759 & - & - \\
\hline Age (ref: 15-24) & & & 0.065 & & - \\
\hline $25-44$ & $35.8(2.4)$ & $1.48(0.88,2.49)$ & & - & \\
\hline $45-64$ & $42.2(2.5)$ & $1.94(1.08,3.49)$ & & - & \\
\hline $65+$ & $38.4(3.0)$ & $1.65(0.84,3.24)$ & & - & \\
\hline Education (ref: <primary) & & & 0.128 & & - \\
\hline$<$ Secondary & $40.2(2.8)$ & $1.30(1.04,1.63)$ & & - & \\
\hline Secondary & $37.6(2.5)$ & $1.17(0.89,1.53)$ & & - & \\
\hline College & $36.2(3.4)$ & $1.10(0.79,1.53)$ & & - & \\
\hline Employment (ref: unemployed) & & & 0.616 & & - \\
\hline AFF & $37.6(3.6)$ & $0.80(0.54,1.19)$ & & - & \\
\hline Transportation & $35.2(4.0)$ & $0.73(0.48,1.11)$ & & - & \\
\hline Business/service & $37.1(3.1)$ & $0.79(0.51,1.21)$ & & - & \\
\hline Other & $36.7(2.8)$ & $0.77(0.52,1.16)$ & & - & \\
\hline Rural residence (ref: urban) & $38.6(2.7)$ & $1.12(0.83,1.50)$ & 0.463 & - & - \\
\hline Region (ref: South-West) & & & 0.188 & & - \\
\hline North & $37.6(2.5)$ & $1.31(0.81,2.12)$ & & - & \\
\hline North-East & $44.5(4.4)$ & $1.75(1.00,3.06)$ & & - & \\
\hline East & $34.5(3.8)$ & $1.15(0.66,1.98)$ & & - & \\
\hline Mid-South & $44.6(4.3)$ & $1.75(1.01,3.04)$ & & - & \\
\hline North-West & $41.3(5.5)$ & $1.53(0.82,2.85)$ & & - & \\
\hline
\end{tabular}

$\mathrm{Cl}$ : confidence interval; OR: odds ratio; SHS: secondhand smoke; AFF: agriculture, forestry, fishing

a Sample weights were used to account for clustering and stratification factors

${ }^{b}$ Adjusted for all of the other variables listed in the model

serious illness (AOR 0.84, 95\% CI: 0.60, 1.16, Table $3)$, the belief that smoking causes serious illness (AOR: 1.63, 95\% CI: 1.14, 2.33), rules not allowing smoking at home (AOR: 1.73, 95\% CI: 1.25, 2.40), noticed smoking warnings on billboards (AOR: 1.43 , 95\% CI: 1.03, 1.75), and visited a doctor within the 
past year (AOR: 1.46, 95\% CI: 1.12, 1.90). Interaction terms were not significant at $\mathrm{p}<0.05$.

\section{Quit smoking}

Variables associated with quitting smoking from bivariate analyses at $p<0.10$ included the belief that SHS causes serious illness $(p=0.004)$, the belief that smoking causes serious illness $(p=0.001)$ or heart attacks $(p=0.006)$, home smoking rules $(\mathrm{p}<0.001)$, noticed signs promoting cigarettes $(p=0.004)$, gender $(p=0.005)$, age $(p<0.001)$, education $(\mathrm{p}=0.001)$, employment $(\mathrm{p}<0.001)$, and region $(\mathrm{p}<0.001)$ (Table 4$)$.

Table 4. Quit smoking: unadjusted and adjusted associations ${ }^{a}$ with the belief that secondhand smoke causes serious illness among ever-smokersb, 2010 China Global Adult Tobacco Survey

\begin{tabular}{|c|c|c|c|c|c|}
\hline Characteristic & $\begin{array}{l}\text { Prevalence } \\
\% \text { among } \\
\text { former } \\
\text { smokers } \\
\text { (Sid Err \%) }\end{array}$ & $\begin{array}{l}\text { Unadjusted OR } \\
\qquad\left(95^{\circ} \% \mathrm{CI}\right)\end{array}$ & p-value & $\begin{array}{l}\text { Adjusted }{ }^{b} \text { OR } \\
\left(95^{\circ} \% \text { CI) }\right.\end{array}$ & p-value \\
\hline Believe SHS causes illness (ref: no) & $17.7(1.5)$ & $1.38(1.10,1.73)$ & 0.004 & $1.44(1.15,1.81)$ & 0.002 \\
\hline \multicolumn{6}{|l|}{ Believe smoking causes (ref: no) } \\
\hline Serious illness & $17.2(1.3)$ & $1.66(1.21,2.27)$ & 0.001 & $1.66(1.21,2.28)$ & 0.002 \\
\hline Lung cancer & $16.1(1.2)$ & $1.01(0.77,1.32)$ & 0.946 & - & - \\
\hline Stroke & $17.8(1.9)$ & $1.21(0.93,1.56)$ & 0.148 & - & - \\
\hline Heart attacks & $18.8(1.7)$ & $1.43(1.10,1.85)$ & 0.006 & - & - \\
\hline Smoking not allowed at home (ref: allowed) & $29.1(3.5)$ & $2.57(1.86,3.56)$ & $<0.001$ & $2.71(1.90,3.89)$ & $<0.001$ \\
\hline \multicolumn{6}{|l|}{ Noticed smoking warnings (ref: no) } \\
\hline In newspapers & $16.8(2.1)$ & $1.08(0.78,1.49)$ & 0.661 & - & - \\
\hline On billboards & $15.5(2.0)$ & $0.95(0.69,1.30)$ & 0.732 & - & - \\
\hline On television & $16.4(1.3)$ & $1.04(0.78,1.41)$ & 0.774 & - & - \\
\hline On posters & $14.8(2.3)$ & $0.90(0.62,1.33)$ & 0.599 & - & - \\
\hline On vehicles & $14.0(2.0)$ & $0.82(0.59,1.16)$ & 0.255 & - & - \\
\hline Elsewhere & $15.5(2.7)$ & $0.96(0.65,1.41)$ & 0.817 & - & - \\
\hline Noticed cigarette promotions (ref: no) & $11.4(1.7)$ & $0.62(0.45,0.86)$ & 0.004 & - & - \\
\hline Male (ref: female) & $15.7(1.1)$ & $0.62(0.44,0.87)$ & 0.005 & - & - \\
\hline Age (ref: 15-24) & & & $<0.001$ & & $<0.001$ \\
\hline $25-44$ & $9.9(1.5)$ & $1.06(0.54,2.08)$ & & $1.02(0.54,1.92)$ & \\
\hline $45-64$ & $17.5(1.2)$ & $2.06(0.97,4.38)$ & & $1.92(0.97,3.80)$ & \\
\hline $65+$ & $37.2(2.5)$ & $5.76(3.01,11.03)$ & & $4.72(2.68,8.32)$ & \\
\hline Education (ref: <primary) & & & 0.001 & & - \\
\hline$<$ Secondary & $19.5(1.9)$ & $0.84(0.60,1.19)$ & & - & \\
\hline Secondary & $13.6(1.4)$ & $0.55(0.41,0.74)$ & & - & \\
\hline College & $15.1(2.0)$ & $0.62(0.41,0.93)$ & & - & \\
\hline Employment (ref: unemployed) & & & $<0.001$ & & $<0.001$ \\
\hline AFF & $14.7(1.6)$ & $0.33(0.24,0.46)$ & & $0.60(0.44,0.82)$ & \\
\hline Transportation & $8.0(1.5)$ & $0.17(0.10,0.27)$ & & $0.29(0.17,0.49)$ & \\
\hline Business/service & $10.5(1.7)$ & $0.23(0.16,0.33)$ & & $0.39(0.27,0.57)$ & \\
\hline Other & $16.0(2.8)$ & $0.37(0.24,0.58)$ & & $0.56(0.36,0.89)$ & \\
\hline Rural residence (ref: urban) & $15.4(1.3)$ & $0.90(0.67,1.20)$ & 0.468 & - & - \\
\hline Region (ref: South-West) & & & $<0.001$ & & 0.001 \\
\hline North & $10.9(1.5)$ & $0.86(0.47,1.56)$ & & $1.62(0.87,3.02)$ & \\
\hline North-East & $25.4(3.1)$ & $2.39(1.31,4.36)$ & & $1.52(0.85,2.72)$ & \\
\hline East & $18.0(2.4)$ & $1.54(0.84,2.82)$ & & $0.76(0.38,1.49)$ & \\
\hline Mid-South & $15.5(2.0)$ & $1.29(0.71,2.33)$ & & $2.23(1.23,4.05)$ & \\
\hline North-West & $13.8(3.0)$ & $1.13(0.55,2.31)$ & & $0.88(0.39,1.99)$ & \\
\hline
\end{tabular}


The final model for ever-smokers quitting smoking included the belief that SHS causes serious illness (AOR: $1.44 ; 95 \%$ CI: $1.15,1.81$, Table 4 ), the belief that smoking causes serious illness (AOR: 1.66, 95\% CI: $1.21,2.28)$, and rules not allowing smoking at home (AOR: 2.71, 95\% CI: 1.90, 3.89). The final model also included age $(\mathrm{p}<0.001)$, employment $(\mathrm{p}<0.001)$, and region $(\mathrm{p}=0.001)$ (Table 4$)$. Interaction terms were not significant at $\mathrm{p}<0.05$.

\section{DISCUSSION}

The results of this study demonstrate that current smokers' attitudes about exposure to SHS could play an important role in the overall strategy to reduce tobacco use in China. The aim of this study was to ascertain whether the belief that SHS causes serious illness was associated with intention to quit, attempts to quit, and quitting smoking among Chinese adult smokers. After adjusting for covariates, there was a significant association between the belief that SHS causes serious illness and intention to quit and quitting smoking. But the association between the belief that SHS causes serious illness and attempting to quit smoking did not reach statistical significance. Analyses also indicated significant associations with several covariates: rules about smoking at home, noticed information on billboards about the dangers of smoking, noticed signs promoting cigarettes, visited a doctor within the past year, and the belief that smoking causes lung cancer. The belief that exposure to SHS causes serious illness is an additional element to include in a comprehensive tobacco-control strategy.

The finding that smokers who believe SHS exposure causes serious illness were more likely to intend to quit smoking or quit smoking is consistent with other studies ${ }^{6,19}$. Concern about exposing others, particularly children, to SHS has been cited as one of the strongest indicators for quitting smoking ${ }^{19,20}$. Increasing knowledge of the dangers of SHS also decreases tolerance for smoking at home ${ }^{6,19,20}$. This finding may in part be explained by Chinese culture, which values responsibility to one's family and filial piety respect for one's parents and ancestors ${ }^{11,13}$. China has a highly collectivistic culture in which the needs of the group come before the needs of the individual ${ }^{21}$. If smoking is perceived as a threat to the health of one's family, collectivism may explain a willingness among Chinese smokers to quit smoking in order to protect members of their in-group ${ }^{21,22}$. Social disapproval of smoking is a more significant predictor of regretting smoking in China than in Western countries, which may induce quitting smoking behavior ${ }^{22,23}$. Strong family relationships are also associated with abstaining from smoking in China $^{25}$. Future anti-tobacco campaigns should focus on education about the dangers of exposures to SHS in the context of one's in-group and family ${ }^{25,26}$.

Although only $2.4 \%$ of women smoke in China, $72.4 \%$ are exposed to SHS, with $38.0 \%$ exposed on a daily basis, many of whom do not recognize the dangers of SHS exposure ${ }^{27}$. Consequently, women bear nearly $80 \%$ of the burden of disease due to SHS exposure ${ }^{28}$. Children in China with chronic exposure to SHS have respiratory problems that continue into adulthood, including impaired lung function, coughing, sneezing, and phlegm ${ }^{29}$. Smokefree laws that are strictly enforced have resulted in a significant reduction in SHS exposure in the public places of Guangzhou, Harbin, Shanghai, Shenzhen, and Tiajin $^{30}$. Greater efforts are required to promote smoke-free environments that weaken smoking customs $^{30}$.

After adjusting for covariates, the belief that SHS causes serious illness did not reach statistical significance for attempting to quit smoking. Previous research has demonstrated that health concerns for friends and family are among the primary reasons Chinese adult smokers attempt to quit smoking ${ }^{11,12}$. However, quit attempts are less successful in China among smokers without firsthand experiences of the adverse health effects of smoking tobacco, personally or within the family ${ }^{11,12}$. Education must also include outreach to smokers living alone and who may not feel the onus of protecting family and friends.

The finding that adult smokers who did not allow smoking at home were 2.46 times more likely to quit smoking is in accord with published data ${ }^{31,32}$. Homesmoking bans have proven to be powerful smoking interventions: they reduce the likelihood of being a current smoker, reduce the number of cigarettes consumed per day, and increase the number of quit attempts $^{31}$. In China, concern regarding the health effects of SHS exposure was the greatest reason for not allowing smoking at home, but most families still allow smoking in at least one room ${ }^{2,32}$. Current laws 
in China ban smoking in selected outdoor locations and all indoor public places, including gymnasiums, libraries, museums, trains, classrooms, dorms, and schools $^{2}$. There is a paucity of information about penalties for violations of these regulations however, and there is no national ban on smoking in the workplace in China ${ }^{2}$.

The result that smokers who noticed information about the dangers of smoking on billboards were more likely to have attempted to quit also aligns with the literature, which demonstrates that mass media campaigns are effective tobacco-control methods ${ }^{33,34}$. Of the media analyzed that displayed smoking, only billboards reached statistical significance for attempted to quit. Visually explicit advertisements are particularly successful for increasing quit attempts $^{34}$. Billboards displaying adverse health outcomes of smoking increase public awareness of smoking dangers and decrease smoking prevalence ${ }^{33}$. Billboards may also include Quitline service information, which has shown promise as a smoking cessation service in Hong Kong ${ }^{35}$.

The results further suggest that adult smokers who believe smoking causes serious illness were more likely to have intended or attempted to quit, which is in accord with other studies ${ }^{25,26}$. Most former smokers cite health concerns as the primary motivation for quitting smoking, but the perceived risks and harms of smoking are low in China compared to other countries ${ }^{25,26}$. Among Chinese smokers, the greatest factor for attempting and intending to quit is knowledge of the adverse health effects of smoking, but misconceptions regarding health consequences of smoking remain pervasive among Chinese smokers ${ }^{11,12}$. Educational campaigns to improve public perceptions of smoking risks, combined with social support and accessible smoking cessation clinics, are effective strategies to increase quit attempts ${ }^{11,12,36}$.

Noticing signs promoting cigarettes was not significantly associated in this study with quitting smoking behavior, and numerous studies have demonstrated that tobacco advertisements foster positive attitudes about tobacco use ${ }^{37,38}$. At the time GATS was conducted in 2010, China did not have laws or regulations for restricting the advertising or promoting of tobacco products ${ }^{2,38}$. Since GATS was conducted, a subsequent law banned the use of signs and mass media to advertise or promote cigarettes ${ }^{2}$. The challenge is to ensure that these new regulations are enforced ${ }^{2}$.

Smokers who attempted to quit smoking were more likely to have visited a doctor within the past year, which also agrees with the literature ${ }^{20,39}$. Simple advice or brief interventions from nonsmoking physicians have been shown to be a cost-effective means to increase quit attempts and quit rates ${ }^{20,39}$. Larger interventions and pharmacotherapy are even more successful for smokers with high nicotine dependence in China ${ }^{39,40}$. However, this message may be muted by virtue of the fact that $46.7 \%$ of male physicians in China have been reported as active smokers $^{41}$.

\section{Limitations}

The findings of this study are subject to limitations. The use of cross-sectional survey data limits our ability to make causal inferences between the exposure variables and quitting smoking behavior. Furthermore, the overall prevalence of smoking has likely been under represented as roughly 200 million Chinese workers, who are known to have a disproportionately high number of smokers compared to the rest of the country, work and live in temporary accommodation away from their registered household ${ }^{2}$. Another limitation is that GATS data include only one smoker per household, which precludes the analysis of the influence of other smokers on smoking proclivity ${ }^{2}$. Other survey limitations include self-reporting and recall biases ${ }^{2}$.

\section{CONCLUSIONS}

The results of this study contribute an additional significant tenet to a comprehensive tobaccocontrol strategy, namely, the belief that exposure to SHS causes serious illness. This message can be incorporated into the other, established control methods like explicit warning labels, high taxes, physician advice, and mass media campaigns, to help decrease the overall burden of tobacco use.

\section{REFERENCES}

1. Koplan J, Eriksen M. Smoking cessation for Chinese men and prevention for women. Lancet. 2015;386(10002):14223. doi:10.1016/s0140-6736(15)00416-x

2. Yang GH. Global Adult Tobacco Survey (GATS) China 2010 Country Report. China Three Gorges Publishing 
House. 2011.

3. Chen Z, Peto R, Zhou M, Iona A, Smith M, Yang L, et al. Contrasting male and female trends in tobacco-attributed mortality in China: evidence from successive nationwide prospective cohort studies. Lancet. 2015;386(10002):144756. doi: 10.1016/s0140-6736(15)00340-2

4. Liang X. Report of China City Adult Tobacco Survey 2013-14. 2015. CDC Foundation, Atlanta, Georgia, USA.

5. Öberg M, Jaakkola MS, Woodward A, Peruga A, PrüssUstün A. Worldwide burden of disease from exposure to second-hand smoke: a retrospective analysis of data from 192 countries. Lancet. 2011;377(9760):139-46. doi: 10.1016/s0140-6736(10)61388-8

6. Australian Institute of Health and Welfare. 2007 National Drug Strategy Household Survey: detailed findings. AIHW cat. no. PHE 107 (Drug Statistics Series no. 22) Canberra: AIHW. 2008: www.aihw.gov.au/publications/ index.cfm/title/10674. Accessed 15 October 2016.

7. Kruger J, Patel R, Kegler M, Babb SD, King BA. Perceptions of harm from secondhand smoke exposure among US adults, 2009-2010. Tob Induc Dis. 2016;14(1):3. doi: 10.1186/s12971-016-0069-8

8. World Health Organization. Tobacco in China. 2016. http://www.wpro.who.int/china/mediacentre/ factsheets/tobacco/en/. Accessed 16 October 2016.

9. Campaign for Tobacco Free Kids. Tobacco Burden Facts: China. 2016. http://global.tobaccofreekids.org/files/ pdfs/en/China_tob_burden_en.pdf. Accessed 11 October 2016.

10. Yang T, Jiang S, Barnett R, Peng S, Yu L. Individual and city-level determinants of secondhand smoke exposure in China. Int J Health Geogr. 2015;14(1):36.

doi: 10.1186/s12942-015-0029-1

11.Jiang Y, Elton-Marshall T, Fong GT, Li Q. Quitting smoking in China: findings from the ITC China Survey. Tob Control. 2010;19(Suppl 2):i12-7.

doi: $10.1136 /$ tc.2009.031179

12.Feng G, Jiang Y, Li Q, Yong HH, Elton-Marshall T, Yang J, et al. Individual-level factors associated with intentions to quit smoking among adult smokers in six cities of China: findings from the ITC China Survey. Tob Control. 2010;19(Suppl 2):i6-11. doi: 10.1136/tc.2010.037093

13. Yang T, Fisher KJ, Li F, Danaher BG. Attitudes to smoking cessation and triggers to relapse among Chinese male smokers. BMC Public Health. 2006;6(1):65. doi: 10.1186/1471-2458-6-65

14. World Health Organization. Global Adult Tobacco Survey (GATS); 2016. http://www.who.int/tobacco/ surveillance/survey/gats/en/. Accessed 3 October 2016.

15. Palipudi KM, Morton J, Hsia J, Andes L, Asma S, Talley B, et al. Methodology of the Global Adult Tobacco Survey-2008-2010. Glob Health Promot. 2016;23(Suppl 2):3-23. doi: 10.1177/1757975913499800

16. Winickoff JP, Friebely J, Tanski SE, Sherrod C, Matt
GE, Hovell MF, et al. Beliefs about the health effects of "thirdhand" smoke and home smoking bans. Pediatrics. 2009;123(1):e74-9. doi: 10.1542/peds.2008-2184

17. Ma GX, Tan Y, Fang CY, Toubbeh JI, Shive SE. Knowledge, attitudes and behavior regarding secondhand smoke among Asian Americans. Prev Med. 2005;41(2):446-53. doi: 10.1016/j.ypmed.2004.11.022

18. Dormann CF, Elith J, Bacher S, Buchmann C, Carl G, Carré $\mathrm{G}$, et al. Collinearity: a review of methods to deal with it and a simulation study evaluating their performance. Ecography. 2013 Jan 1;36(1):27-46.

19. Song AV, Glantz SA, Halpern-Felsher BL. Perceptions of second-hand smoke risks predict future adolescent smoking initiation. J Adolescent Health. 2009;45(6):61825. doi: 10.1016/j.jadohealth.2009.04.022

20.Tong EK, Ong MK, Vittinghoff E, Pérez-Stable EJ. Nondaily smokers should be asked and advised to quit. Am J Prev Med. 2006;30(1):23-30. doi: 10.1016/j.amepre.2005.08.048

21. Mao A, Bottorff JL, Oliffe JL, Sarbit G, Kelly MT. A Qualitative Study on Chinese Canadian Male Immigrants' Perspectives on Stopping Smoking: Implications for Tobacco Control in China. Am J Men's Health. 2016; 19:1557988316644050. doi: 10.1177/1557988316644050

22.Im PK, McNeill A, Thompson ME, Fong GT, Xu S, Quah AC, et al. Individual and interpersonal triggers to quit smoking in China: a cross-sectional analysis. Tob Control. 2015;24(Suppl 4):iv40-7.

doi: 10.1136/tobaccocontrol-2014-052198

23. Sansone N, Fong GT, Lee WB, Laux FL, Sirirassamee $\mathrm{B}$, Seo HG, et al. Comparing the experience of regret and its predictors among smokers in four Asian countries: findings from the ITC surveys in Thailand, South Korea, Malaysia, and China. Nicotine Tob Res. 2013;15(10):1663-72. doi: 10.1093/ntr/ntt032

24. Shakib S, Zheng H, Johnson CA, Chen X, Sun P, Palmer $\mathrm{PH}$, et al. Family characteristics and smoking among urban and rural adolescents living in China. Prev Med. 2005;40(1):83-91. doi: 10.1016/j.ypmed.2004.05.029

25. Gallus S, Muttarak R, Franchi M, Pacifici R, Colombo P, Boffetta P, et al. Why do smokers quit? Eur J Cancer Prev. 2013;22(1):96-101. doi:10.1097/cej.0b013e3283552da8

26. McCaul KD, Hockemeyer JR, Johnson RJ, Zetocha K, Quinlan K, Glasgow RE. Motivation to quit using cigarettes: a review. Addict Behav. 2006;31(1):42-56. 10.1016/j.addbeh.2005.04.004

27. Gong X, Luo X, Ling L. Prevalence and Associated Factors of Secondhand Smoke Exposure among Internal Chinese Migrant Women of Reproductive Age: Evidence from China's Labor-Force Dynamic Survey. International J Environ Res Pub He. 2016 Apr 1;13(4):371. doi: 10.3390/ijerph13040371

28. Gan Q, Smith KR, Hammond SK, Hu TW. Disease burden of adult lung cancer and ischaemic heart disease from passive tobacco smoking in China. Tob Control. 2007 
Dec 1;16(6):417-22. doi: 10.1136/tc.2007.021477

29. He QQ, Wong TW, Du L, Jiang ZQ, Yu TS, Qiu H, et al. Environmental tobacco smoke exposure and Chinese schoolchildren's respiratory health: a prospective cohort study. Am J Prev Med. 2011 Nov 30;41(5):487-93. doi: 10.1016/j.amepre.2011.07.019

30. World Health Organization. Smoke-free policies in China: evidence of effectiveness and implications for action. 2015 Oct 1.

31. Mills AL, Messer K, Gilpin EA, Pierce JP. The effect of smoke-free homes on adult smoking behavior: a review. Nicotine Tob Res. 2009;ntp122. doi: 10.1093/ntr/ntp122

32.Zheng P, Kegler MC, Berg CJ, Fu W, Wang J, Zhou X, et al. Correlates of smoke-free home policies in Shanghai, China. BioMed Res Int. 2014. doi: 10.1155/2014/249534

33. Liu H, Tan W. The effect of anti-smoking media campaign on smoking behavior: The California experience. Ann Econ Financ. 2009;10(1):29-47.

34. Durkin SJ, Biener L, Wakefield MA. Effects of different types of antismoking ads on reducing disparities in smoking cessation among socioeconomic subgroups. Am J Public Health. 2009;99(12):2217-23.

doi: 10.2105 /ajph.2009.161638

35. Abdullah AS, Lam TH, Chan SS, Hedley AJ. Which smokers use the smoking cessation Quitline in Hong Kong, and how effective is the Quitline?. Tob Control. 2004 Dec 1;13(4):415-21. doi: 10.1136/tc.2003.006460

36. Liu S, Zhang M, Yang L, Li Y, Wang L, Huang Z, et al. Prevalence and patterns of tobacco smoking among Chinese adult men and women: findings of the 2010 national smoking survey. J Epidemiol Commun Health. 2017 Feb 1;71(2):154-61. doi: 10.1136/jech-2016-207805

37. Yang T, Rockett IR, Li M, Xu X, Gu Y. Tobacco advertising, environmental smoking bans, and smoking in Chinese urban areas. Drug Alcohol Depen. 2012;124(1):121-7. doi: 10.1016/j.drugalcdep.2011.12.021

38. Li L, Yong HH, Borland R, Fong GT, Thompson ME, Jiang Y, et al. Reported awareness of tobacco advertising and promotion in China compared to Thailand, Australia and the USA. Tob Control. 2009;18(3):222-7.

doi: $10.1136 /$ tc.2008.027037

39. Stead LF, Bergson G, Lancaster T. Physician advice for smoking cessation. Cochrane Database Syst Rev. 2008;2(2). doi: 10.1002/14651858.cd000165.pub2

40.Kim SS, Chen W, Kolodziej M, Wang X, Wang VJ, Ziedonis D. A systematic review of smoking cessation intervention studies in China. Nicotine Tob Res. 2012 Jan 16;14(8):891-9. doi: 10.1093/ntr/ntr289

41. Smith DR, Zhao I, Wang L. Tobacco smoking among doctors in mainland China: a study from Shandong province and review of the literature. Tob Induc Dis. 2012;10(1):14. doi: 10.1186/1617-9625-10-14

ACKNOWLEDGEMENTS

The author appreciates the encouragement, assistance and advice from Dr. Thomas Novotny, MD, MPH, Deputy Assistant Secretary for Health (Science and Medicine), U.S. Department of Health \& Human Services.

CONFICT OF INTEREST Author has completed and submitted the ICMJE Form for Disclosure of Potential Conflicts of Interest and none was reported.

\section{FUNDING}

There was no source of funding for this research.

PROVENANCE AND PEER REVIEW

Not commissioned; externally peer reviewed 